\title{
Three-dimensional Topology-Independent Methods to Look for Global Topology
}

\author{
Boudewijn F. Roukema ${ }^{1,2}$ and Vincent Blanloeil ${ }^{3}$ \\ ${ }^{1}$ Observatoire de Strasbourg, 11, rue de l'Université, Strasbourg F-67000, France \\ ${ }^{2}$ Nicolaus Copernicus Astronomical Center, ul. Bartycka 18, 00-716 Warsaw, Poland \\ ${ }^{3}$ Institut de recherche mathématique avancée, Université Louis Pasteur et CNRS, \\ 7 rue René-Descartes, F-67084 Strasbourg Cedex, France \\ roukema@iap.fr,blanloei@math.u-strasbg.fr
}

\begin{abstract}
The space-like hypersurface of the Universe at the present cosmological time is a three-dimensional manifold. A non-trivial global topology of this space-like hypersurface would imply that the apparently observable universe (the sphere of particle horizon radius) could contain several images of the single, physical Universe. Recent three-dimensional techniques for constraining and/or detecting this topology are reviewed. Initial applications of these techniques using X-ray bright clusters of galaxies and quasars imply (weak) candidates for a non-trivial topology.
\end{abstract}

\section{Introduction}

If the physical Universe is smaller than the "observable Universe", i.e., if the fundamental polyhedron of the Universe is smaller than the sphere of horizon radius in the universal covering space, then some (or many) regions of space will be observable at several (or many) different "look-back" times de Sitter 1917; Lemaitre 1958). The word "Universe" can be taken here to refer either theoretically to the space-like hypersurface at the present cosmological time, or observationally to the observed past time cone considered in comoving coordinates. Since space-like hypersurfaces are three-dimensional, use of three-dimensional information on astrophysical objects known to exist in the covering space provides a straight-forward way to search for or constrain the global topology of the Universe.

The reader is referred to Lachièze-Rey \& Luminet (1995) and to other contributions to this workshop for an introduction to cosmological topology and to Luminet (1998) for an interesting historical introduction.

A brief mathematical description of the rela- tionship between a universal covering space $X$, a compact 3-manifold $M$ and its fundamental polyhedron $P$ is provided in $\$ 2$. For a fuller introduction to three-dimensional geometry and topology, see Thurston $(1982,1997)$.

Three-dimensional topology detecting techniques are based on the required existence of multiple topological images of single physical objects. Techniques applicable to objects observed to successively larger scales are described in $\$ 4.1$ ("cosmic crystallography", Lehoucq et al. 1996), \$1.2 ("brightest X-ray clusters", Roukema \& Edge 1997) and $\$ 4.3$ ("local isometry detection", Roukema 1996).

A Friedmann-Lemaître-Robertson-Walker metric (implying constant curvature of any spatial hypersurface) is assumed throughout this paper. Comoving coordinates are used, i.e., positions of objects observed in our past time cone are projected to the (3-D) spacelike hypersurface at the present epoch, $t=t_{0}$. Spectroscopic redshifts, denoted $z$, are used to obtain radial distance estimates (termed "proper distances" by Weinberg 1972, eq.14.2.21 in the $t=t_{0}$ space-like hypersurface.

\section{Topology of Compact 3-Manifolds: Covering Spaces and Fundamental Polyhedra}

If we assume the Universe to have an FLRW metric and a trivial topology, then it is one of the three 3manifolds $S^{3}$, the 3 -sphere, $E^{3}$, Euclidean 3 -space, or $H^{3}$, the hyperbolic 3 -space (negatively curved). This

\footnotetext{
${ }^{1}$ The "proper distance" should not be confused with the quantity that Weinberg (1972, p.485) calls "proper motion distance" and that Peebles (1993, p.321, eq.13.36) calls "angular size distance". The proper distance and proper motion distance are identical for zero curvature, but not otherwise.
} 
apparent space is called the "universal covering space", which we call $X$.

This can be related to the real 3-manifold of the Universe, which we call $M$ and which can be thought of physically in terms of a fundamental polyhedron $P$ as follows.

One can construct a complete geometric 3-manifold by choosing $\Gamma$, a discrete subgroup of the group of isometries of $X$, acting freely on $X$ (i.e., the set $\{g \in$ $\Gamma: g x=x\}$ is trivial for all $x$ in $X)$ and take the quotient $X / \Gamma$. Of course, at the present we know neither $X$ nor $\Gamma$ for the real Universe.

Observations favour $X=E^{3}\left[\Omega_{0}+\lambda_{0}=1\right.$ in terms of standard metric parameters, where $\lambda_{0} \equiv$ $\left.\Lambda c^{2} /\left(3 H_{0}^{2}\right)\right]$ and $X=H^{3}\left(\Omega_{0}+\lambda_{0}<1\right)$, though as long as $\Omega_{0}+\lambda_{0}=1$ remains reconcilable with observations, $X=S^{3}$ is likely to remain a possibility where $\Omega_{0}+\lambda_{0}=1+\epsilon$ and $\epsilon \ll 1$ if $\Omega_{0}$ and $\lambda_{0}$ are only measured by traditional, local physical properties such as density. [An interesting global method is the effect predicted by Barrow et al. (1985, p937), who showed that compactness and a small amount of rotation of the observable Universe could in principle enable the case $\Omega_{0}+\lambda_{0}=1$ to be distinguished from the case $\Omega_{0}+\lambda_{0}=1 \pm \epsilon,(\epsilon \ll 1)$ by the presence of a "spiralling effect" which could be seen in the cosmological microwave background.]

Then, a convex fundamental polyhedron for the discrete group $\Gamma$ of the group of isometries of $X$ is a convex polyhedron $P$ in $X$ such that its interior $P^{\circ}$ fulfils:

i) the members of $\left\{g P^{\circ}: g \in \Gamma\right\}$ are mutually disjoint,

ii) $X=\cup\{g P: g \in \Gamma\}$

iii) $\{g P: g \in \Gamma\}$ is a locally finite family of subsets of $X$.

To find a fundamental polyhedron, for all $g \neq 1$ in $\Gamma$ and for $x$ in $X$, we define $H_{g}(x)=\{y \in X$ : $d(x, y)<d(x, g y)\}$. Then the Dirichlet domain $D(x)$, with center $x$, for $\Gamma$ is: $D(x)=\cap\left\{H_{g}(x): g \neq 1 \in \Gamma\right\}$ when $\Gamma$ is non trivial and $D(x)=X$ when $\Gamma$ is trivial The closure $\bar{D}(x)$ is a convex fundamental polyhedron for $\Gamma$.

Using a fundamental polyhedron $P$ in $X$, one can build a 3-manifold $M$ by gluing together the sides of $P$. Poincaré's fundamental polyhedron theorem proves that the inclusion of $P$ in $X$ induces an isometry from $M$ to $X / \Gamma$, where $\Gamma$ is the discrete subgroup of the group of isometries of $X$ such that $(S, R)$ is a group presentation for $\Gamma$ with $S$ the set of sides of $P$ and $R$ the set of relations determined by the gluing.

When $\Gamma$ is not trivial this construction gives nontrivial topology.

Let us be more precise. Choose a base point $x_{0}$ of $X$ and let $a: S^{1} \rightarrow X / \Gamma$ be a loop based at $x_{0}$; let $b:[0,1] \rightarrow X$ be a lift of $a$ starting at $x_{0}$ and ending at $g_{a} x_{0}$ (note that $g_{a}$ is unique since $\Gamma$ acts freely). The map $l: \pi_{1}(X / \Gamma) \rightarrow \Gamma$ defined by $l(a)=g_{a}$ is a homomorphism which is obviously surjective (i.e., onto). Suppose for $a \in \pi_{1}(X / \Gamma)$ we have $l(a)=1$. Then a lift of $a$ in $\pi_{1}(X)$ is equal to 1 since $X$ is simply connected, hence $a=1$ and $l$ is injective (i.e., 1:1). Therefore, $l: \pi_{1}(X / \Gamma) \rightarrow \Gamma$ is an isomorphism.

Since $\Gamma$ is a non-trivial group, the fundamental group $\pi_{1}(X / \Gamma)$, which could be thought of as the group of non-shrinkable loops of $X / \Gamma$, and hence of $M$, is nontrivial. That is, $M$ has a non-trivial topology and can be referred to as multi-connected.

For an example of theoretical ideas for the physical meaning of $\Gamma$, see e Costa \& Fagundes (1998). Here we merely consider observational detection of $\Gamma$.

Characterising the "size" of fundamental polyhedra of 3-manifolds in a way useful observationally requires at least two parameters. Here we adopt the "injectivity radius", $r_{\text {inj }}$, i.e., half of the smallest distance from an object to one of its topological images; and the outradius, $r_{+}$, which is the radius of the smallest sphere (in the covering space) which totally includes the fundamental polyhedron (Cornish et al. 1998a). We refer here to $2 r_{\text {inj }}$ as the injectivity diameter and $2 r_{+}$as the out-diameter. For a discussion of these and related size parameters, see Cornish et al. (1998a), and note that $2 r_{\text {inj }}$ and $2 r_{+}$are similar to the parameters $\alpha$ and $\beta$ adopted by Lachièze-Rey \& Luminet (1995, §10.3.3).

While both parameters represent in some sense the "size" of the fundamental polyhedron, it should particularly be remembered that many hyperbolic compact 3 -D manifolds can have $r_{\text {inj }} \ll r_{+}$. Since we live in the plane of a disc galaxy - which obscures most astronomical observations within several degrees of the plane - it would be difficult to measure $2 r_{\text {inj }}$ if it's the size of a geodesic at an angle "close" to the Galactic plane.

\section{Multiple topological images of observable objects}

In a multi-connected universe, the covering space, or "apparent" universe, is tiled by copies of the fundamental polyhedron (Dirichlet domain). So the basic principle of detecting multi-connectedness is to find multiple "topological images". 7

In a flat covering space, the particle horizon radius, $R_{H}$, is not geometrically constrained to $r_{\text {inj }}$ and $r_{+}$. In a hyperbolic covering space, the two quantities are both

\footnotetext{
${ }^{2}$ Also called "topological clones". The terminology "ghosts" is not preferred since it implies that some images are less physically real than others.
} 
directly related to the curvature radius, $R_{C}$, (at least in the orientable cases), by Mostow's rigidity theorem which states that a homotopy equivalence between two orientable hyperbolic 3-manifolds is homotopic to an isometry. Hence, $r_{\text {inj }}$ and $r_{+}$are bounded below by $R_{C}$ and hence by $R_{H}$ to within a few orders of magnitude (depending on which of the many compact hyperbolic manifolds applies to the universe).

So, if physical conditions in the very early Universe (quantum epoch) tend to minimise the volume and the "present-day" Universe is negatively curved, then the fundamental polyhedron may well be small enough that multiple topological images of points of space are likely to exist within the present-day observable sphere. Zero curvature provides no such constraint.

Since the Universe is of finite age, information (photons) can only be received from within a sphere (in the covering space) of finite radius around the observer. This sphere may contain several copies of the fundamental polyhedron, in which multiple topological images of single physical objects can be found. However, objects which are seen further towards this "horizon" are seen earlier in the history of the Universe, so are seen at different stages of the transition between a relatively smooth (to $\sim 0.001 \%$ ) material to the formation of high-density objects such as quasars, clusters of galaxies and galaxies. So any set of observable objects can only be seen in a certain sub-sphere (or a spherical shell) which is smaller than the observable sphere.

Depending on the metric parameters $\left(\Omega_{0}, \lambda_{0}\right)$ of the Universe, the fraction of the comoving observable sphere covered by a set of observable objects varies.

Fig. 1 shows some characteristic distances to which different types of objects have so far been observed in significant quantities for a range of the metric parameters covering the values consistent with a wide range of observational cosmological tests. The redshifts used are indicative only.

The proper distance to a redshift $z$ can be evaluated in general as

$$
d(z)=\frac{c}{H_{0}} \int_{1 /(1+z)}^{1} \frac{\mathrm{d} a}{a \sqrt{\Omega_{0} / a-\kappa_{0}+\lambda_{0} a^{2}}}
$$

where $\kappa_{0} \equiv \Omega_{0}+\lambda_{0}-1$. 3 This can be expressed in terms of the curvature radius

$$
R_{C} \equiv \frac{c}{H_{0} \sqrt{\left|\kappa_{0}\right|}}
$$

and the proper motion distance $d_{p m}(z)$ as

$$
d(z)= \begin{cases}R_{C} \sinh ^{-1}\left[d_{p m}(z) / R_{C}\right], & \kappa_{0}<0 \\ d_{p m}(z), & \kappa_{0}=0 \\ R_{C} \sin ^{-1}\left[d_{p m}(z) / R_{C}\right], & \kappa_{0}>0 .\end{cases}
$$

\footnotetext{
${ }^{3}$ For readers of the popular Peebles (1993), $\Omega_{0}, \lambda_{0}$ and $\kappa_{0}$ correspond to Peebles' $\Omega, \Omega_{\Lambda}$ and $-\Omega_{R}$ respectively.
}
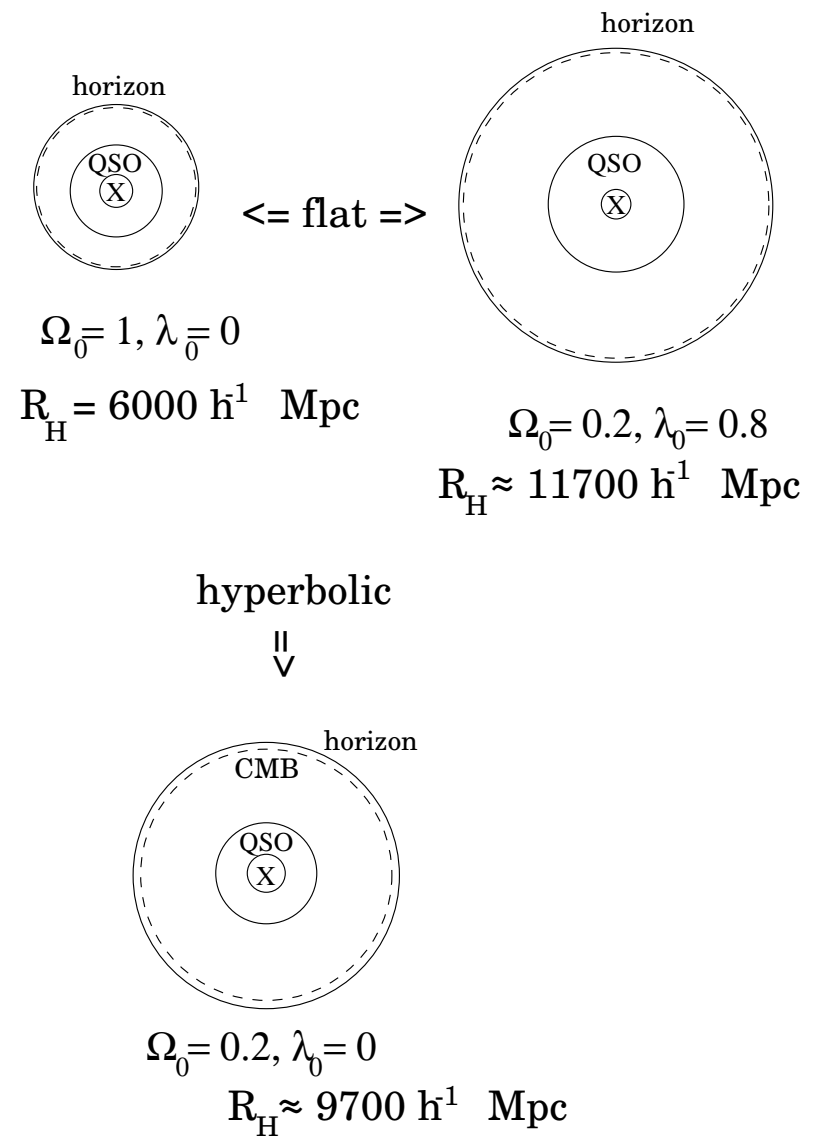

Figure 1: Relative sizes of observable domains of the universal covering space, depending on different options for the metric parameters $\Omega_{0}, \lambda_{0}$, shown in comoving coordinates on a radially linear (proper distance) scale. The horizon radius $R_{H}$ is defined by the age of the Universe. The inner circles for each choice of metric indicate from the centre outwards redshifts of: $z=0.5$ (roughly that to which we can see X-ray emission from the richest clusters of galaxies); $z=3$ (to which there is a high quasar density); and $z=1000$ (roughly the redshift of the cosmic microwave background, dashed circle). In the flat models, the tangential distance scale is constant and the same as the radial scales; in the hyperbolic model, the tangential distance scale increases (more Gpc per mm on the page) as a function of increasing radius. 
If $\Omega_{0}>0$ and $\lambda_{0}=0$, then the closed expression

$$
d_{p m}(z)=\frac{c}{H_{0}} \frac{2\left[z \Omega_{0}+\left(\Omega_{0}-2\right)\left(\sqrt{\Omega_{0} z+1}-1\right)\right]}{\Omega_{0}^{2}(1+z)}
$$

can be used (Weinberg 1972, p.485). Or, for $\kappa_{0} \neq 0$, equations (11) and (2) can be combined to give

$$
\frac{d(z)}{R_{C}}=\sqrt{\left|\kappa_{0}\right|} \int_{1 /(1+z)}^{1} \frac{\mathrm{d} a}{a \sqrt{\Omega_{0} / a-\kappa_{0}+\lambda_{0} a^{2}}}
$$

Only very bright galaxy clusters are seen to $z=0.5$, and systematic "all-sky" surveys for galaxy clusters [e.g., to an X-ray luminosity of $L_{X}(0 \cdot 1-$ $\left.2 \cdot 4 \mathrm{keV}) \gtrsim 10^{45} \mathrm{erg} / \mathrm{s}\right]$ are only presently being carried out to $z \sim 0 \cdot 1$. Quasars are in fact seen to redshifts higher than $z \approx 3$, but drop quickly in number density (e.g., Shaver et al. 1998).

The fraction of the horizon distance covered by the sphere to $z=3$ only decreases slightly between the models with different metric parameters, from $50 \%$ in the $\Omega_{0}=1, \lambda_{0}=0$ (Einstein-de Sitter) model to $39 \%$ in the $\Omega_{0}=0 \cdot 2, \lambda_{0}=0$ model. If multiple copies of the fundamental polyhedron are to be detected in a radial direction, the relative efficiencies of 3-D methods using objects (e.g., quasars) visible to these redshifts does not change much. Due to the negative curvature, however, the number of copies of a fundamental polyhedron which could be placed alongside one another in a tangential direction around a sphere makes a search to $z \lesssim 3$ much less efficient relative to a CMB (cosmic microwave background) search for an $\Omega_{0}=0 \cdot 2, \lambda_{0}=0$ universe relative to an Einstein-de Sitter universe.

Therefore, if the Universe is as negatively curved as to give $\Omega_{0}=0 \cdot 2, \lambda_{0}=0$, then 3 -D searches for topological images are going to be most efficient for multiconnected manifolds which have $r_{\text {inj }} \ll r_{+}$. Since this is the case for many of the hyperbolic compact manifolds, a 3-D method which can be applied to objects seen to $z \lesssim 3$ may be capable of detecting non-trivial topology of the Universe, i.e., at least one of the generators $g \in \Gamma$, in this case.

On the other hand, the flat universe model dominated by a cosmological constant $\left(\Omega_{0}=0 \cdot 2, \lambda_{0}=0 \cdot 8\right)$ shows nearly identical relative efficiencies of $z \lesssim 3$ and CMB methods as for the flat, $\lambda_{0}=0$ model, but the relative usefulness of objects seen to $z \lesssim 0.5$ is much lower than in the model with a cosmological constant than in the model without.

\section{Previous 3-D constraints and new 3-D methods}

The out-diameter, $2 r_{+}$, is strongly bounded below by about $60 h^{-1} \mathrm{Mpc}$ to $150 h^{-1} \mathrm{Mpc}$ by the ab- sence of secondary topological images of the Coma cluster of galaxies (Gott 1980) and by the existence of "large scale structure" ("great walls" and filaments formed by galaxies, de Lapparent et al. 1986; Geller \& Huchra 1989; da Costa et al. 1993; Deng et al. 1996; Einasto et al. 1997). Equivalently, this is a constraint that $2 r_{+} \gtrsim R_{H} / 100$. Although mathematically not strictly excluded, it would seem difficult for the injectivity diameter, $2 r_{\text {inj }}$, to be as small as $2 r_{\mathrm{inj}} \lesssim R_{H} / 100$ in a way that would fit the spatial distribution and physical properties of observed objects.

At distances greater than $R_{H} / 10$, the formation and evolution of astrophysical objects becomes much more serious than at small scales.

In addition, catalogues of observed objects are limited to either wide-angle surveys to small radial distances, or "deep" surveys over small solid angles. The two types of surveys have complementary advantages for detecting topology, though the use of the wide-angle surveys is simpler. The increase in the characteristic radial distances and solid angular areas of these surveys will increase rapidly over the next few decades.

To avoid the problems of evolution, methods based primarily on the $3-\mathrm{D}$ positions of the objects (rather than their physical properties) are needed. The methods of "cosmic crystallography" (\$4.1) and of "local isometry searching" (4.3) were created for use in catalogues of objects which are subject to evolutionary effects on the individual astrophysical objects. If the evolutionary effects are not too strong (and if viewing angle is not a problem), the former method is applicable. If many objects have only a subset of their topological images visible due to such effects, the latter method is necessary.

Nevertheless, the existence of a "unique" object (e.g., much more brilliant than all others of its class) at a large radial distance could still be useful in finding a lower bound to $r_{+}$(particularly if a systematic survey over $4 \pi$ steradians were available). This idea can be used by consideration of the "richest" galaxy cluster found by the X-ray emission emitted by its hot $T \sim 10^{7} \mathrm{~K}$ gas $(\$ 4.2$ ).

For simplicity, most of the discussion below is presented in the context of a flat, $\Omega_{0}=1, \lambda_{0}=0$ universe, but it should be kept in mind that physical (Cornish et al. 1996) and geometrical arguments favour a hyperbolic universe. For detailed discussions of the hyperbolic case, see the work of Fagundes (1985; $1989 ; 1996)$. For numerical representations of compact hyperbolic manifolds and visualisation software, SNAPPEA and GeOMview (http://www.geom.umn.edu/) are recommended.

For completeness, it should also be mentioned 
that attempts have been made to use the essentially two-dimensional information in the CMB to observationally bound $r_{\text {inj }}$ from below, by making assumptions on the distributions of amplitudes and phases of temperature fluctuations at the epoch of the CMB, by accepting foreground corrections as valid and by considering either particular cases of flat geometries (Stevens et al. 1993; Starobinsky 1993; Jing \& Fang 1994; de Oliveira Costa \& Smoot 1995; Levin et al. 1998) or individual cases of hyperbolic geometries (Bond et al. 1998). If the caveats of these techniques are accepted as correct, then $r_{\text {inj }}$ seems not much smaller than the horizon size. Indeed, the latter authors find a candidate hyperbolic manifold, for $\Omega_{0}=0 \cdot 8$, which is "preferable to standard CDM" relative to the observed CMB (Bond et al., §4.3), but the volume of its fundamental polyhedron is slightly larger than that of the observable sphere.

It should also be noted that non-trivial topology is usually adopted in $N$-body simulations of the formation of galaxies and large-scale structure, but for numerical rather than physical reasons (see Farrar \& Melott 1990 for an explicit analysis).

\subsection{Cosmic crystallography}

In a catalogue of objects in which multiple topological images of single physical objects are often seen, a histogram of object-object pair separations [for all $N(N+1) / 2$ pairs in a set of $N$ objects] should show sharp peaks due to pairs of topological images separated by multiples of the vectors which generate the fundamental polyhedron from the covering space. Lehoucq et al. (1996) used simulations to show that this method should be efficient and independent of topology, at least for the case of zero curvature. The application of this method to the classical Abell and ACO cluster catalogues, to $z \approx 0.25$, didn't show any obvious topological signal (but see also Fagundes \& Gausmann 1997). The method was devised to also work in the cases of non-zero curvature, but its practical application under astronomical conditions has yet to be carried out.

The Abell and ACO catalogues will soon be superceded by X-ray selected "all-sky" '4 catalogues which suffer less serious systematic biases, but only to $z \sim 0 \cdot 1$ in programs already in progress for finding rich clusters.

The intrinsically brightest of these clusters should be found to a factor of several higher in redshift, though in numbers too small for the histogram method to show any peaks. In this case, the following method can be

\footnotetext{
4 "All-sky" can mean as little as $2 / 3$, though usually more, of $4 \pi$ steradians, due to obscuration by the Galaxy.
}

applied.

\subsection{X-ray clusters as "standard can- dles"}

If a small number of the brightest objects of a given class (in particular, galaxy clusters selected in X-rays) are known out to a given redshift, these can be considered to be unique objects if their evolutionary properties are simple enough.

In the case of the richest galaxy clusters, which are dominated by hot gas, these objects are unlikely to become any less luminous as time increases, though they are likely to increase somewhat in luminosity. This is because galaxy clusters are the largest gravitationally bound objects which have had time to collapse in the age of the Universe, and are dominated by their hot hydrogen gas which is in approximate kinetic equilibrium. Within cosmologically available time scales, it is difficult to see how a high enough fraction of this gas could either cool, escape or turn into galaxies in order for a secondary topological image at a lower redshift (i.e., at a more recent epoch) to be invisible in X-rays.

Roukema \& Edge (1997) noticed that
RX J1347.5-1147, which is probably the brightest Xray cluster known (in the $0 \cdot 1-2 \cdot 4 \mathrm{keV}$ frequency band) is quite distant from us (with respect to other known galaxy clusters). So, if we could be sure that there were no topological images of this cluster closer to us in any direction, then the distance to this cluster would give the lower limit $r_{+} \gtrsim 1100 \pm 100 h^{-1} \mathrm{Mpc}$. (The uncertainty is due to the observational uncertainty in the metric parameters; the range shown in Fig. 1 is adopted.) However, since galactic obscuration is important, this is strictly speaking a weaker limit, i.e., $2 r_{+} \gtrsim 1100 \pm 100 h^{-1} \mathrm{Mpc}$.

Because the object is unique, the only topological image pair geodesics which are excluded are those extending from the known image to the borders of the observed volume. Small closed geodesics in other directions, e.g., roughly perpendicular to any large geodesic running from RX J1347.5-1147 to a distant point in the north galactic cone defined by $b^{I I}>20^{\circ}, z \leq 0 \cdot 451$, would not contradict the existence of RX J1347.5-1147 as an (apparently) unique object. Hence, this method only constrains $2 r_{+}$rather than $2 r_{\text {inj }}$.

Serendipitously, a candidate for the topology was noticed by comparing the $3-\mathrm{D}$ positions of the several bright clusters listed by Roukema \& Edge (1997). Three (of the seven clusters studied) form a right angle (to $2 \%$ accuracy) with side lengths equal within $1 \%$. This is just what would be expected in the case of a $T^{2} \times X$ manifold where $X$ is unknown. There is no obvious physical motivation for this case to be favoured, 
though it is commonly used for pedagogical purposes, as in several of the early attempts at trying to constrain topology using the $\mathrm{CMB}$ as observed by the COBE satellite.

Roukema \& Edge (1997) list several arguments against topological identity of the three clusters, but the cleanest observational test would be to verify or refute the existence of further implied topological images. While further implied topological images could exist at high redshifts, the cluster might not have formed at those early epochs. So implied images at low enough redshifts that the cluster is guaranteed to be in existence with a minimum luminosity should be considered.

Specific predictions of this (weak) candidate 3manifold containing two known generators $g_{1}, g_{2}$ and one unknown generator $g_{3}$ are as follows. If this candidate as suggested by Roukema \& Edge (1997) were correct, then the object seen by ROSAT, RX J203150.4 -403656 , should be a galaxy cluster at $0.38<z<0 \cdot 40$, and the Arp-Madore galaxy cluster AM $0750-490$ (which would be the low redshift topological image of MS $1054-0321$ ) would be at $0 \cdot 23<z<0 \cdot 26$.

\subsection{Local isometry searches}

The previous methods can only be applied to objects whose evolution is relatively simple. The objects which can be easily seen to $z \sim 3$ in significant numbers, quasars, have evolutionary properties which are not well understood. They are likely to have short lifetimes, either with recurrent bursts related to (maybe) mergers of galaxy dark matter haloes or differing lifetimes depending on individual quasar properties. In addition, according to the "unified model" of active galactic nuclei (AGN), a quasar seen from a very different angle appears much fainter, as a Seyfert galaxy, for example, with a very small chance of having already been observed in high-redshift galaxy searches.

One approach to using quasars is to consider special cases. Fagundes \& Wichoski (1987) searched for images of the Galaxy seen as quasars in directions separated by $180^{\circ}$ or $90^{\circ}$.

A more general method is to accept the problem of multiple topological image visibility and search in a large catalogue for the rare cases in which several objects in two different topological images of a single physical 3-D region are both visible. In other words, one searches for an isometry between two regions of the covering space, each of a few hundred $h^{-1} \mathrm{Mpc}$ in size.

If several such isometries are found, then these should be used to generate the full set of transformations from the covering space to the fundamental polyhedron. These would then be confirmed (or refuted) by the predictions of the 3-D positions of multiple topological images of other quasars, or by comparison with the CMB.

This method was first presented by Roukema (1996) and applied to a catalogue of $N \approx 5000$ quasars at $z>$ 1. Two isometries, i.e., two pairs of quasar quintuplets separated by more than $300 h^{-1} \mathrm{Mpc}$, were found. Due to the number density distribution of quasars, this is not necessarily due to topological imaging. Simulated catalogues showed that there is about a $30 \%$ chance of finding two similar coincidences in a universe of trivial topology with the same observational selection criteria for finding quasars.

At best, these two isometries could be considered as defining a weak candidate for the 3-manifold in which we live. This candidate would be non-orientable.

The technique as presented by Roukema (1996) was only an initial implementation of the basic principle. The parameters chosen may not necessarily be optimal for obtaining a detection.

For example, although the number of isometries of $n$-tuplets is higher when $n$ is lower, it may well be possible that the signal (due to topological isometries) may increase faster than the noise (due to the number density distribution of the catalogue for a simply connected universe) when $n$ is lowered, so that the signal-to-noise ratio would increase.

Another use of the technique using the same size data set would be to test a series of universe models with incrementally different metric parameters (e.g., $\left.\Omega_{0}=0 \cdot 20,0 \cdot 21,0 \cdot 22, \ldots, 1 \cdot 10\right)$, in particular the negatively curved models. The increment should be chosen as a function of the uncertainty regarding quasars' (3D) positions.

Alternatively, one could allow for a scaling factor when comparing $n$-tuplets, in which case isometries due to any value of the curvature would automatically be detected. However, this would also increase the number of chance coincidences of $n$-tuplets, and is not strictly correct - it would distort the local geometrical relations if the $n$-tuplets are not small enough.

In the method of adopting successive values of the metric parameters, the successive signal-to-noise ratios (numbers of isometric $n$-tuplet pairs due to topology compared to numbers due to chance) are $S_{i} / N_{i}$ where

$$
S_{i} \begin{cases}=0, & \left(i \not i^{*}\right) \\ >0, & \left(i \approx i^{*}\right)\end{cases}
$$

and $\left(\Omega_{0}, \lambda_{0}\right)_{i *}$ are the metric parameters of the real Universe. The signal-to-noise ratio $S_{i} / N_{i}$ is then zero except when the metric parameters are close to correct, i.e., $i \approx i^{*}$, and has a maximum of $S_{i^{*}} / N_{i^{*}}$. (The values of the metric parameters would then be quite tightly constrained.) 
If a scaling factor is used instead, then the calculation should be much faster (since a single set of metric parameters is adopted), but the signal-to-noise ratio becomes

$$
\begin{aligned}
\frac{\sum_{i} S_{i}}{\sum_{i} N_{i}+\sum_{i \neq j} N_{i j}} & =\frac{\sum_{i \approx i^{*}} S_{i}}{\sum_{i} N_{i}+\sum_{i \neq j} N_{i j}} \\
& \approx \sum_{i *} N_{i}+\sum_{i \neq j} N_{i j} \\
& \ll S_{i^{*}} / N_{i^{*}},
\end{aligned}
$$

where $N_{i j}$ are numbers of chance coincidences for scaling factors which imply inconsistent curvature estimates. So, the signal-to-noise ratio is much lower if scaling is used. Even if the scaling is done requiring consistent values of the curvature between $n$-tuplets at different redshifts (which would probably slow down the calculation), the signal-to-noise ratio would still be lower than for the successive metric value method. Additionally, distortions due to the greater than zero size of $n$-tuplets would make $S_{i^{*}}$ in the scaling method slightly lower than for the successive metric method.

The other development of this method is that since quasars are visible to about $R_{H} / 2$, future quasar surveys will provide a more thorough sampling of this volume, so that the "rare" cases searched for will increase in number and the chances of detection (if the topology is detectable) will increase.

\section{Conclusion}

Several methods have been developed in the last few years to either detect or constrain the topology of the spatial part of the Universe. The relative efficiency (in terms of fundamental polyhedron crossings) of 3$\mathrm{D}$ to 2-D methods depends moderately on the precise values of the metric parameters $\Omega_{0}, \lambda_{0}$. Objects seen to about $z \sim 3$ would cross half the horizon distance for any presently accepted metric parameters, and in a cosmological constant dominated universe, objects seen to $z \sim 0.1-0.5$ would cover many fewer copies of the fundamental polyhedron than the CMB.

Initial applications of 3-D methods to existing observational catalogues or individual observations indicate several (weak) candidates for the 3-manifold in which we live. (Or more precisely, for some of the generators of the 3-manifold.) These candidates are falsifiable with moderate observational investment in telescope time.

Moreover, further development of the local isometry search method is presently possible for application to existing observational quasar catalogues.

Looking to the future, new catalogues of objects made over the next few years, in particular all-sky surveys of quasars, will possibly allow the topology of the
Universe to be detected to a high significance by the local isometry search method. Alternatively, the "circles method" of Cornish et al. (1998b) applied to the observations by either MAP or Planck (planned CMB satellites) is likely to either reveal or constrain the topology of the Universe.

Within a decade, we should know whether or not the topology of the Universe is detectable, and if so what it is.

This research has been partially supported by the Polish Council for Scientific Research Grant KBN 2 P03D 00813.

\section{References}

Barrow, J.D., Juskiewicz, R., Sonoda, D.H., 1985, M.N.R.A.S., 213, 917

Bond, J.R., Pogosyan, D., Souradeep, T., 1998, Class.Quant.Grav, this vol, astro-ph/9804041

Cornish, N.J., Spergel, D.N., Starkman, G.D., 1996, Phys.Rev.Lett., 77, 215

Cornish, N.J., Spergel, D.N., Starkman, G.D., 1998a, astro-ph/9708225

Cornish, N.J., Spergel, D.N., Starkman, G.D., 1998b, astro-ph/9801212

da Costa, L.N., in Cosmic Velocity Fields, ed. Bouchet, F., Lachièze-Rey, M., (Gif-sur-Yvette, France: Editions Frontières), p475

de Lapparent, V., Geller, M.J., Huchra, J.P., 1986, Ap.J., 302, L1

Deng, X.-F., Deng, Z.-G., Xia, X.-Y., 1996, Chin.Astron.Astroph., 20, 383

de Oliveira Costa, A., Smoot, G.F., 1995, Ap.J., 448, 477

de Sitter, W., 1917, M.N.R.A.S., 78, 3

e Costa, S.S., Fagundes, H.V., 1998, astro$\mathrm{ph} / 9801066$

Einasto, J., et al., 1997, Nature, 385, 139

Fagundes, H.V., 1985, Ap.J., 291， 450

Fagundes, H.V., 1989, Ap.J., 338, 618

Fagundes, H.V., 1996, Ap.J., 470, 43

Fagundes, H.V., Gausmann, E., 1997, astro$\mathrm{ph} / 9704259$

Fagundes, H.V., Wichoski, U.F., 1987, Ap.J., 322, L5 
Farrar, K. \& Melott, A.L., 1990, Computers in Physics, 4, 185

Geller, M.J., Huchra, J.P., 1989, Science, 246, 897

Gott, J.R.I., 1980, M.N.R.A.S., 193, 153

Jing, Y.-P., Fang, L.-Z., 1994, astro-ph/9409072

Lachièze-Rey, M., Luminet, J.-P., 1995, PhysRep, 254, 136

Lehoucq, R., Luminet, J.-P., Lachièze-Rey, M., 1996, A.\&A., 313, 339

Lemaître, G., 1958, in La Structure et l'Evolution de l'Univers, Onzième Conseil de Physique Solvay, ed. Stoops, R., (Brussels: Stoops), p1

Levin, J., Scannapieco, E., Silk, J., 1998, astro$\mathrm{ph} / 9802021$

Luminet, J.-P., 1998, Conceptions de l'espace en physique, Ecole des Houches, Acta Cosmologica

Peebles, P.J.E., 1993, Principles of Physical Cosmology, Princeton, U.S.A.: Princeton Univ. Press

Roukema, B.F., 1996, M.N.R.A.S., 283, 1147 astro$\mathrm{ph} / 9603052$ )

Roukema, B.F., Edge, A.C., 1997, M.N.R.A.S., 292, 105 astro-ph/9706166)

Shaver, P.A., Hook, I.M., Jackson, C.A., Wall, J.V., Kellermann, K.I., 1998, astro-ph/9801211

Starobinsky, A.A., 1993, JETPLett, 57, 622

Stevens, D., Scott, D., Silk, J., 1993, PhysRevLett, 71,20

Thurston, W.P., 1982, Bull.Am.Math.Soc., 6, 357

Thurston, W.P., 1997, Three-Dimensional Geometry and Topology, ed. Levy, S., Princeton, U.S.A.: Princeton University Press

Weinberg, S., 1972, Gravitation and Cosmology, New York, U.S.A.: Wiley 